Bifurcations of Snap-back Repellers with application to Border-Collision Bifurcations

Glendinning, Paul

2009

MIMS EPrint: 2009.35

Manchester Institute for Mathematical Sciences

School of Mathematics

The University of Manchester

\footnotetext{
Reports available from: http://eprints.maths.manchester.ac.uk/

And by contacting: The MIMS Secretary

School of Mathematics

The University of Manchester

Manchester, M13 9PL, UK
} 


\title{
BIFURCATIONS OF SNAP-BACK REPELLERS WITH APPLICATION TO BORDER-COLLISION BIFURCATIONS
}

\author{
PAUL GLENDINNING \\ SCHOOL OF MATHEMATICS \\ AND \\ $C I C A D A$ \\ (CENTRE FOR INTERDISCIPLINARY COMPUTATIONAL AND DYNAMICAL ANALYSIS) \\ UNIVERSITY OF MANCHESTER \\ OXFORD ROAD, MANCHESTER M13 9PL \\ UNITED KINGDOM. \\ E-MAIL: P.A.GLENDINNING@MANCHESTER.AC.UK
}

\begin{abstract}
The bifurcation theory of snap-back repellers in hybrid dynamical systems is developed. Infinite sequences of bifurcations are shown to arise due to the creation of snap-back repellers in non-invertible maps. These are analogous to the cascades of bifurcations known to occur close to homoclinic tangencies for diffeomorphisms. The theoretical results are illustrated with reference to bifurcations in the normal form for border-collision bifurcations.
\end{abstract}

Keywords: snap-back-repeller, hybrid system, border-collision bifurcation.

\section{INTRODUCTION}

One of the few methods available to prove that a map has chaotic behaviour is to show the existence of a homoclinic orbit, i.e. a fixed point (or equivalently by looking at a higher iterate, a periodic orbit) which another orbit approaches in both forwards and backwards time. This implies the existence of a 'homoclinic tangle' of the stable and unstable manifolds of the fixed point, and associated unstable chaos. In parameterized families of maps, homoclinic orbits are typically created (as the parameter varies) by the development of a homoclinic tangency: at some critical parameter the stable and unstable manifolds of a fixed point intersect tangentially, and on one side of this critical value there are no intersections, and hence no homoclinic orbit to the fixed point, and on the other side of this critical value there are two transversal intersections $[5,6,8]$. Associated with such a tangency there are sequences of bifurcations which create the orbits which must exist once the stable and unstable manifolds of

Date: May 8, 2009. 
the fixed point intersect transversely, and in low dimensional cases these are wellunderstood; it is even possible to show that strange attractors are created during this process. The general picture for the simplest periodic orbits (those that pass once through a neighbourhood of the fixed point in each period) is that they are created in a sequence of saddle-node bifurcations which accumulate on the parameter at which the tangency exists $[4,6]$.

These results rely on the existence of stable and unstable manifolds for the fixed point; it must be a saddle. If the fixed point is repelling, so all the eigenvalues of the Jacobian matrix lie outside the unit circle, then there is no local stable manifold and so, at least for invertible maps, there can be no homoclinic orbits. This is not true of non-invertible maps. In this latter case, the fixed point can have more than one preimage and so it is possible that there exists a point (other then the fixed point) which maps to the fixed point and such that a sequence of preimages can be chosen which tend to the fixed point in reverse time. Thus there can be a homoclinic orbit to the fixed point even though it has no local stable manifold. Under some technical assumptions, such a homoclinic orbit implies the existence of an unstable chaotic set, and this type of homoclinic orbit for a non-invertible map is often referred to as a snap-back repeller. Although there are many papers describing the dynamics associated with snap-back repellers (and even some controversy as to the best definitions to use) and their natural generalizations to heteroclinic loops, there is no analysis of how snap-back repellers are created, nor a description of the bifurcations creating the unstable invariant set which must exist.

The aim of this paper is to analyze a codimension one bifurcation by which a snap-back repeller is created, and to look at the subsidiary bifurcations associated with this. In particular, it will be shown that an infinite cascade of more complicated snap-back repellers are created, and that this sequence may be on one side of the fundamental bifurcation, or on both sides. This infinite cascade is in many ways analogous to the cascades of bifurcations in the standard homoclinic case $[4,6]$. To do this we define conditions which ensure the persistence of a snap-back repeller in a family of maps, and then show how the dynamics comes into being as a function of parameters if the persistence conditions are not satisfied. To some extent this clarifies the standard definition of Marotto [12,13], which is persistent, from the conditions of Lin et al [11] or Shi and Yu [15], which can include non-persistent cases.

Many of the examples of snap-back repellers described in the literature are somewhat contrived, but non-invertible maps are becoming more and more relevant to engineering applications through their appearance in hybrid systems. Here, the interaction between continuous and discrete variables leads naturally to nonsmooth systems and non-invertibility. To illustrate the relevance of these results the normal form for border collision bifurcations will analyzed. Indeed, this is such a good motivating example, and makes some of the technical issues so much clearer, that we will 
start with a brief analysis of the creation of a snap-back repeller in this normal form before giving the formal definitions for the general case.

Section three then deals with definitions and persistence of snap-back repellers, and section four contains the main bifurcation results, both for periodic points and for more complicated snap-back repellers. In section five we return to the example, showing how the results apply to this case and confirming the natural scaling results for the cascade of bifurcations.

\section{BORDER COLLISION BIFURCATIONS}

Border collision bifurcations occur in piecewise smooth systems with discrete time. Phase space is divided into two regions by a switching surface $\Sigma$; in each of the regions, labelled $L$ and $R$ (for left and right) the dynamics is determined by smooth maps $f_{\alpha}, \alpha=L, R$, and the equations are continuous but not differentiable across $\Sigma$. In applications the switching surface can be thought of as some threshold above which a different control strategy comes into play, or a different physical model is more appropriate.

The simplest bifurcations of such systems which cannot occur in smooth systems involves a fixed point (or periodic orbit) on the switching surface $\Sigma$. The two-dimensional normal form for this bifurcation was derived in [1,14], and if the switching surface is transformed to be the $y$-axis $(x=0)$ then the local evolution with $\mathbf{x}=(x, y)^{T}$ is

$$
\mathbf{x}_{n+1}=\left\{\begin{array}{lll}
A_{L} \mathbf{x}+\mathbf{m} & \text { if } & x \leq 0 \\
A_{R} \mathbf{x}+\mathbf{m} & \text { if } & x \geq 0
\end{array}\right.
$$

where the matrices $A_{L}$ and $A_{R}$, and the vector $\mathbf{m}$ are defined as

$$
A_{\alpha}=\left(\begin{array}{cc}
T_{\alpha} & 1 \\
-D_{\alpha} & 0
\end{array}\right), \text { and } \mathbf{m}=\left(\begin{array}{c}
\mu \\
0
\end{array}\right)
$$

for $\alpha=L, R$. The constants $T_{\alpha}$ and $D_{\alpha}$ are the trace and determinant of the Jacobian of the defining equations on the left and right of $\Sigma$, whilst $\mu$ is the bifurcation parameter. If $\mu=0$ then the origin is a fixed point, and this is clearly in $\Sigma$. The question for bifurcation theory is what happens close to the origin when $|\mu|$ is small.

If $\left|D_{R}\right|$ and $\left|D_{L}\right|$ are both less than one then this is a fairly classical situation, which has been treated in a number of papers $[1,2,14]$. More recently the unstable case has been considered and the existence of snap-back repellers has been proved for suitable parameter values [7].

The fixed points of the maps are given by

$$
x_{*}^{\alpha}=\frac{\mu}{1-T_{\alpha}+D_{\alpha}}, \quad y_{*}^{\alpha}=-D_{\alpha} x_{*}^{\alpha}, \quad \alpha=L, R
$$


and $\mathbf{x}_{*}^{R}$ exists provided $x_{*}^{R}>0$, with a similar inequality for the existence of $\mathbf{x}_{*}^{L}$. Given $T_{\alpha}$ and $D_{\alpha}$ these inequalities define the sign of $\mu$ for which these fixed points exist. The fixed points in $x \geq 0$ and $x \leq 0$ coincide at the origin (on $\Sigma$ ) if $\mu=0$. To get interesting dynamics we assume that the signs of $D_{R}$ and $D_{L}$ are different (since $y_{n+1}=-D_{\alpha} x_{n}$ the images of the left and right half planes overlap if the in this case, but not if the signs are the same). We shall assume that $D_{R}$ is positive and $D_{L}$ is negative.

Glendinning and Wong [7] assume further that the eigenvalues of $A_{R}$ are real and distinct and greater than one, so $\mathbf{x}_{*}^{R}$ is an unstable node. This corresponds to the additional conditions

$$
D_{R}>1, \quad T_{R}>2, \quad T_{R}^{2}>4 D_{R}, \quad 1-T_{R}+D_{R}>0
$$

which implies that the fixed point in $x>0$ given by (3) exists if $\mu>0$ and the images of the half planes $x>0$ and $x<0$ lie in the lower half plane. Since $y_{*}^{R}<0$ the fixed point in $x>0$ has a preimage in $x<0$ and this point, $\mathbf{x}_{0}^{L}=\left(x_{0}, y_{0}\right)$, is given by

$$
x_{0}=\frac{D_{R}}{D_{L}} x_{*}^{R}, y_{0}=\frac{1}{D_{L}}\left(T_{R} D_{L}-T_{L} D_{R}-D_{L} D_{R}\right) x_{*}^{R}
$$

If this point is itself to have a preimage in $x>0$ (with a set of preimages converging on the fixed point) then we need $y_{0}<0$. So a necessary condition for the existence of a snap-back repeller is

$$
T_{R} D_{L}-T_{L} D_{R}-D_{L} D_{R} \geq 0 .
$$

Glendinning and Wong [7] analyze the conditions which ensure that there is a snapback repeller which implies chaos, and in particular give sufficient conditions for the preimage of $\left(x_{0}, y_{0}\right)$ in $x>0$ to tend to the fixed point $\left(x_{*}^{R}, y_{*}^{R}\right)$ in backwards time without leaving $x>0$. In the context of the present paper, the real question is what happens if $\mu>0$ (in which case, by rescaling we may assume $\mu=1$ if we wish) and $T_{R} D_{L}-T_{L} D_{R}-D_{L} D_{R}$ changes sign, so we use this expression as a bifurcation parameter.

If

$$
T_{R} D_{L}-T_{L} D_{R}-D_{L} D_{R}=0
$$

then the preimage of $\left(x_{*}^{R}, y_{*}^{R}\right)$ in $x<0$ is

$$
\left(\frac{D_{R}}{D_{L}} x_{*}^{R}, 0\right)
$$

and the preimage of this point under the map in $x>0$ is $\left(x_{1}, y_{1}\right)$ where

$$
x_{1}=0, \quad y_{1}=\frac{1}{D_{L}}\left(D_{R}-D_{L}-D_{R} D_{L}+T_{R} D_{L}\right) x_{*}^{R} .
$$

Since $y_{1}=\left(D_{R} x_{*}^{R} / D_{L}\right)-\mu$ it is always negative provided the fixed point in $x>0$ exists, and in particular, has preimages in $x>0$. The local structure of the map 
in $x>0$ is determined by the eigenvalues and eigenvectors of the linear part of the map. The eigenvalues are the roots of

$$
s^{2}-T_{R} s+D_{R}=0
$$

which, by assumption (4) has two real solutions $\lambda_{ \pm}$with $1<\lambda_{-}<\lambda_{+}$. The corresponding eigenvectors are lines

$$
y=-\frac{D_{R}}{\lambda_{ \pm}}\left(x-x_{*}^{R}\right)-y_{*}^{R}
$$

and so both have negative slope with the eigenvector of $\lambda_{-}$below that of $\lambda_{+}$in $x>x_{*}^{R}$. The approach of the solution through $\left(x_{1}, y_{1}\right)$ to the fixed point in reverse time is determined by its position relative to the intersections of the lines (11) with the $y$-axis. These are the points $\left(0, y_{ \pm}\right)$with

$$
y_{ \pm}=-D_{R}\left(1-\frac{1}{\lambda_{ \pm}}\right) x_{*}^{R}
$$

So, if $y_{+}<y_{1}<y_{-}$then backwards iterates tend to the fixed point in $x>0$ tangential to the branch of the eigenvector of $\lambda_{-}$above and to the left of the fixed point, whilst if $y_{1}<y_{+}$then the accumulation is to the right and below the fixed point. We shall return to these conditions in more detail below.

Now consider a family of disjoint closed (but with non-empty interior) neighbourhoods of the preimages of $\left(x_{1}, y_{1}\right)$ in $x>0$, chosen so that they map onto each other. The image of these sets maps to a region around $\left(x_{1}, y_{1}\right)$ part of which lies in $x>0$ and part in $x<0$. Both of these sets will be mapped to $y<0$ close to $\left(x_{0}, y_{0}\right)$, i.e. the image is 'folded over' and $\left(x_{0}, y_{0}\right)$ will be on the boundary of the image. In the standard argument for the existence of chaos in a snap-back repeller neighbourhoods can be chosen to map over the original sets in a small neighbourhood of the fixed point in $x>0$ allowing a symbolic description of orbits using established techniques. If the preimage is on the boundary of the set, however, there is a chance that the image of this set does not contain a countable number of the preimages which converge on the fixed point, making it impossible to argue for chaotic solutions.

To understand the geometry of the image of a neighbourhood of $\left(x_{0}, y_{0}\right)$ in $y<0$ at the bifurcation point (recall that here $y_{0}=0$ ) we consider the image of a half-disc of radius $\varepsilon$ and with boundary

$$
x_{0}-\varepsilon \leq x \leq x_{0}+\varepsilon, \quad y=0
$$

and

$$
\left(x_{0}+\varepsilon \cos \theta, y_{0}-\varepsilon \sin \theta\right), \quad 0 \leq \theta \leq \pi
$$

for some small $\varepsilon>0$ as shown in Figure 2. The image of the straight line through $\left(x_{0}, y_{0}\right)$ is the line

$$
y-y_{*}^{R}=-\frac{D_{L}}{T_{L}}\left(x-x_{*}^{R}\right)
$$




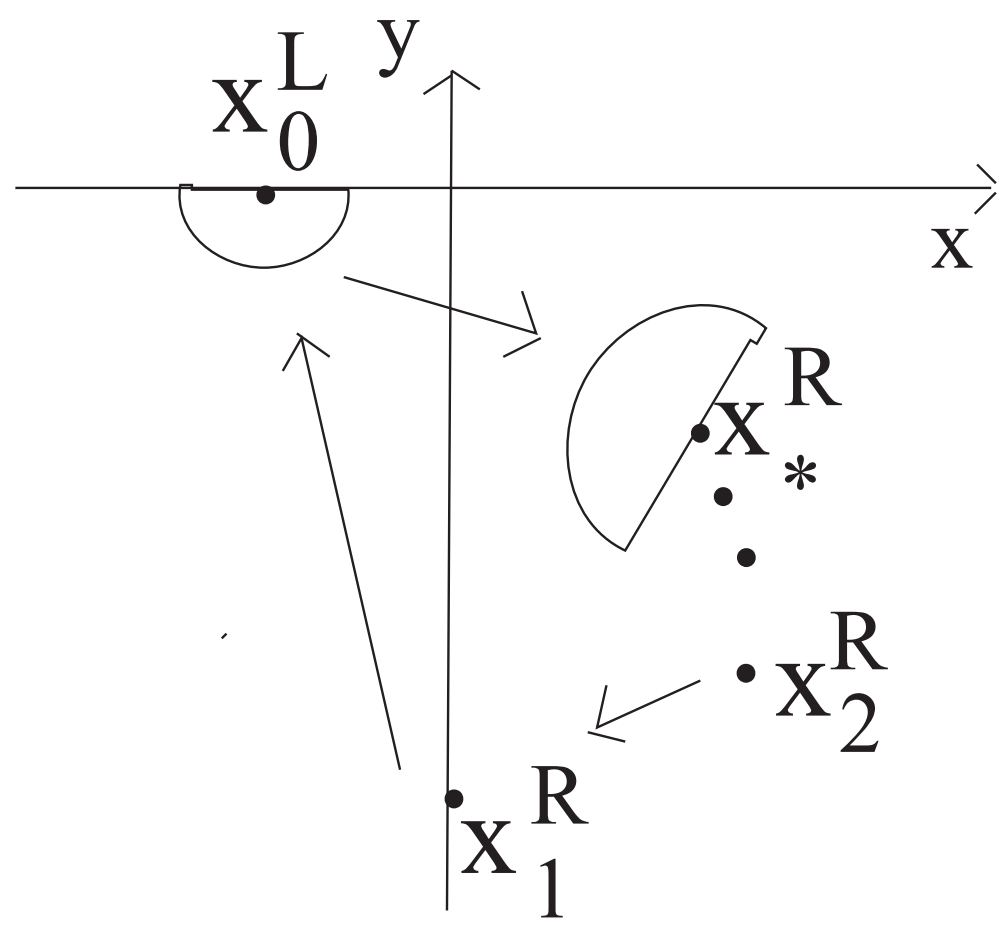

FIGURE 1. Schematic diagram of phase space at the bifurcation point with $\mathbf{x}_{0}^{L}=\left(x_{0}, 0\right), \mathbf{x}_{1}^{R}=\left(0, y_{1}\right), F\left(\mathbf{x}_{0}^{L}\right)=\mathbf{x}_{*}^{R}$ and $F\left(\mathbf{x}_{1}^{R}\right)=\mathbf{x}_{0}^{L}$. The half disc with $\mathbf{x}_{0}^{L}$ on its boundary maps to the half disc with $\mathbf{x}_{*}^{R}$ on its boundary, which in this case is on the opposite side of the fixed point from the preimages of $\mathbf{x}_{1}^{R}$.

which has positive slope if $T_{L}>0$ and negative slope if $T_{L}<0$, whilst the image of the semi-circle is

$$
\left(\begin{array}{l}
x \\
y
\end{array}\right)=\left(\begin{array}{c}
x_{*}^{R} \\
y_{*}^{R}
\end{array}\right)+\varepsilon\left(\begin{array}{c}
T_{L} \cos \theta-\sin \theta \\
-D_{L} \cos \theta
\end{array}\right)
$$

It is useful to see the straight line (13) as $\left(x_{0}+\varepsilon \cos \theta, 0\right), 0 \leq \theta \leq \pi$ for comparison with the semi-circle. In this formulation the image of the line segment is

$$
\left(\begin{array}{l}
x \\
y
\end{array}\right)=\left(\begin{array}{c}
x_{*}^{R} \\
y_{*}^{R}
\end{array}\right)+\varepsilon\left(\begin{array}{c}
T_{L} \cos \theta \\
-D_{L} \cos \theta
\end{array}\right)
$$

and comparison with (16) shows that at the same value of $y$, the image of the semicircle is to the left of the line. Hence if the gradient of the line is greater than (i.e. more positive than) the gradient of the eigenvector corresponding to $\lambda_{-}$the region will contain preimages accumulating on the fixed point in $x>0$ tangential to the branch of the eigenvector of $\lambda_{-}$above and to the left of the fixed point, whilst if the 
gradient is more negative then it will contain the accumulation to the right and below the fixed point.

Four different cases arise depending on whether preimages of $\left(x_{0}, y_{0}\right)$ accumulate on the fixed point from above or below, and depending on whether the image of a half-disc containing $\left(x_{0}, y_{0}\right)$ contains this accumulation or not. To determine the conditions, and which, if any, can be realized, the conditions alluded to in the preceding paragraphs need to be given explicitly.

First let us return to the question of which branch of the unstable eigenvector of $\lambda_{-}$ the backwards iterates of $\left(x_{1}, y_{1}\right)$ converge on. Recall that it is the left hand branch if $y_{+}<y_{1}<y_{-}$, and the right hand branch if $y_{1}<y_{+}$.

Thus convergence is on the left branch (above and to the left of the fixed point) if

$$
\left(1-\frac{1}{\lambda_{-}}\right)<-\frac{D_{R}-D_{L}-D_{R} D_{L}+T_{R} D_{L}}{D_{R} D_{L}}<\left(1-\frac{1}{\lambda_{+}}\right)
$$

whilst convergence is on the right branch if

$$
-\frac{D_{R}-D_{L}-D_{R} D_{L}+T_{R} D_{L}}{D_{R} D_{L}}>\left(1-\frac{1}{\lambda_{+}}\right)
$$

with $\lambda_{ \pm}=\left(T_{R} \pm \sqrt{T_{R}^{2}-4 D_{R}}\right) / 2$. (Note that there are many different ways of writing these equations - the choice here is one which makes it very obvious that all the collected terms are positive, except for the factor of $D_{L}$ which is negative.)

Now, the image of a half-disc in $y \leq 0$ about $\left(x_{0}, y_{0}\right)$ contains part of the left branch of the eigenvector of $\lambda_{-}$if $-D_{L} / T_{L}>-D_{R} / \lambda_{-}$. If $T_{L}>0$ this inequality always holds, whilst if $T_{L}<0$ then the condition is $\lambda_{-}\left|D_{L}\right|<D_{R}\left|T_{L}\right|$; if this condition does not hold, i.e. if

$$
T_{L}<0, \quad \lambda_{-}\left|D_{L}\right|>D_{R}\left|T_{L}\right|
$$

then the image of a half-disc in $y \leq 0$ about $\left(x_{0}, y_{0}\right)$ contains part of the right branch of the eigenvector of $\lambda_{-}$.

Thus if (19) and (20) hold, or if (18) holds but (20) does not hold, then preimages converge to the fixed point on the same side of the fixed point as is covered by the image of a neighbourhood of $\left(x_{0}, y_{0}\right)$ in $y<0$. In this case the standard arguments for the existence of chaos can be made. On the other hand, if these combinations do not hold, the preimages converge on the opposite side of the fixed point as is covered by the neighbourhood of $\left(x_{0}, y_{0}\right)$ and we cannot use standard arguments to create infinitely many recurrent points.

This then, is the situation at the bifurcation point defined by (7), and corresponds to the satisfying or not of condition three in [11]. The aim of the next sections is to give general arguments as to what can happen in general systems near such a bifurcation point. The border-collision normal form will be re-considered in section five in the light of the theoretical results obtained. 


\section{SNAP-BACK REPELLERS AND THEIR PERSISTENCE}

The idea of a snap-back repeller, a natural extension of homoclinic orbits to noninvertible maps, was first considered by Marotto [12] and there has been renewed interest recently in the light of a small technical error in Marotto's original paper (an error which does not change the basic topological argument of the paper $[9,13]$ ) and a desire to apply the idea to more general systems and to more complicated orbits $[3,10,11,15]$. The definitions used below are motivated by a wish to develop a bifurcation theory of snap-back repellers, so the most general definitions are not necessarily the most appropriate for this setting - in particular we want the definition of a regular snap-back repeller to be such that such objects are robust to small perturbations of the system.

Definition 1: hybrid map. A map $F: \mathbb{R}^{n} \rightarrow \mathbb{R}^{n}$ will be called a hybrid map if $\mathbb{R}^{n}$ can be partitioned into a finite number $J$ of disjoint open regions $R_{i}, i=$ $1, \ldots J$, with nonempty interior; and a boundary, $\Sigma$, which is made up of a union of continuously differentiable surfaces which separate these regions: $\mathbb{R}^{n}=\left(\cup_{1}^{J} R_{i}\right) \cup \Sigma$; and $F$ restricted to $R_{i}$ is $C^{2}$. Note that we do not need to impose any continuity conditions for $F$ across $\Sigma$, although the example of border-collision bifurcations in the previous section has continuity of the map (but not of the Jacobian) across the boundary.

Definition 2: regular snap-back repeller. A hybrid map $F: \mathbb{R}^{n} \rightarrow \mathbb{R}^{n}$ has a regular snap-back repeller if there is a fixed point $x^{*} \in R_{k}$ (for some $k$ ) such that all the eigenvalues of $D F\left(x^{*}\right)$ lie outside the unit circle and there is a homoclinic orbit to $x^{*}$ which does not intersect $\Sigma$, i.e. a set of points $\left\{p_{r}\right\}_{0}^{\infty}$ and a map $i: \mathbb{N} \rightarrow\{1, \ldots, J\}$ such that $p_{r} \in R_{i(r)}, F\left(p_{i+1}\right)=p_{i}, i \geq 0, F\left(p_{0}\right)=x^{*}$ and $\lim _{r \rightarrow \infty} p_{r}=x^{*}$ (which implies that there exists $N>0$ such that $p_{r} \in R_{k}$ for all $\left.r>N\right)$. In addition $D F\left(p_{r}\right)$ is non-singular, $r=0,1,2, \ldots$.

This is effectively the definition of regular snap-back repellers of [15], though our assumption of the existence of the homoclinic orbit means we do not need the explicit expansion conditions used there.

The sets $R_{i}$ make it possible to set up a simple symbolic dynamics for orbits which do not intersect $\Sigma$ : let $a(x)=i$ if $x \in R_{i}$ and then define the symbol sequence of $x$, $s(x)$ as

$$
s(x)=a(x) a(F(x)) a\left(F^{2}(x)\right) \ldots
$$

so that the $n^{\text {th }}$ term of the sequence gives the index of the set in which $F^{n-1}(x)$ is contained. Clearly

$$
s \circ F(x)=\sigma \circ s(x)
$$

where $\sigma$ is the standard shift operator on symbol sequences: $\sigma\left(a_{0} a_{1} a_{2} \ldots\right)=a_{1} a_{2} \ldots$ 
Theorem 1. (Marotto) Suppose that a hybrid map $F: \mathbb{R}^{n} \rightarrow \mathbb{R}^{n}$ has a regular snapback repeller with fixed point $x^{*} \in R_{k}$. Then there is an unstable, chaotic invariant set close to the homoclinic orbit. Moreover, there exists $M>0$ and a finite word $A$ of symbols in $\{1, \ldots, J\}$ such that the symbolic description of points in this invariant set contains all sequences of the form

$$
k^{n_{1}} A k^{n_{2}} A k^{n_{3}} A k^{n_{4}} A \ldots, \quad n_{i}>M
$$

and their shifts, and the possibility that $n_{i}=\infty$ is allowed.

Proof: Let $k, N$ and $\left(p_{r}\right)$ be as in the definition of the regular snap-back repeller. Choose a closed neighbourhood of $p_{N+2}, B(N+2)$, which contains $p_{N+2}$ in its interior and no other point $p_{r}$, which is always possible as the $p_{r}$ are disjoint and have a unique accumulation point. By choosing $B(N+2)$ smaller if necessary we can ensure that $B(N+2) \subset R_{k}$ and $B(N+2) \cap F(B(N+2))=\emptyset$ (the former is possible as $R_{k}$ is open, the latter is by continuity of $F$ and the fact that the $p_{r}$ are distinct).

For $m<N+2$ define $B(m)$ inductively by $B(m)=F(B(m+1))$. Reducing the size of the set $B(N+2)$ further if necessary we can assume that $B(m) \subset R_{i(m)}$ for $m=0, \ldots, N+1$ and $B^{\prime}=F(B(0)) \subset R_{k}$. By continuity of $F$ each of the subsets $B(m)$ has $p_{m}$ in its interior, and $x^{*}$ lies in the interior of $B^{\prime}$. Moreover, for $m>N+2$ we can define closed, disjoint sets $B(m) \subset R_{k}$ by $F(B(m))=B(m-1)$; these are disjoint by the condition imposed earlier that $B(N+2) \cap F(B(N+2))=\emptyset$, and converge on $x^{*}$ as $B(m)$ contains the point $p_{m}$ in their interior.

Now, $\operatorname{int}\left(B^{\prime}\right)$ contains $x^{*}$, and hence there exists $M>0$ such that it contains $B(m)$ for all $m>M$. By definition, $B^{\prime}=F^{m+1}(B(m))$ and hence for all $u>M$, $B(u) \subset F^{m+1}(B(m))$ and in particular, there is a close set $B(m, u) \subset B(m)$ such that $F^{m+1}(B(m, u))=B(u)$. More generally, let $m_{1}, \ldots, m_{j}$ be a sequence of integers greater than $M$, then by defining $B\left(m_{1}, \ldots, m_{j}\right) \subset B\left(m_{1}, \ldots, m_{j-1}\right)$ by

$$
F^{m_{1}+1} B\left(m_{1}, \ldots, m_{j}\right)=B\left(m_{2}, \ldots, m_{j}\right)
$$

we have a nested sequence of closed sets, and so

$$
B\left(m_{1}, \ldots, m_{j}, \ldots\right)=\bigcap_{j=1}^{\infty} B\left(m_{1}, \ldots, m_{j}\right)
$$

is non-empty. The symbolic description comes from re-interpreting the indices in terms of a time spent within $R_{k}$ followed by a finite sequence of indices before returning to $B^{\prime}$.

To introduce a parameter $\mu$, we need to describe how the hybrid map, and in particular the partition of $\mathbb{R}^{n}$ of definition 1 , varies as parameters are varied.

Definition 3: continuous family of hybrid maps. Let $\mathcal{M}$ be an open interval. A continuous family of hybrid maps $F: \mathbb{R}^{n} \times \mathcal{M} \rightarrow \mathbb{R}$ is a set of hybrid maps on 
$\mathbb{R}^{n}=\left(\cup_{1}^{J} R_{i}\right) \cup \Sigma$, where this partition is independent of $\mu$, such that the maps $F(x, \mu)$ are hybrid maps for each $\mu \in \mathcal{M}$, the maps $F(x, \mu)$ restricted to $R_{i}$ are $C^{2}$ in their first argument and continuous in the second, and the Jacobian $D F$ of $F$ with respect to $x$ is also continuous in $\mu$.

In other words a continuous family of hybrid maps is a family of hybrid maps such that the maps and their Jacobians with respect to $x$ vary continuously in the parameter $\mu$ on each of the sets $R_{i}$. We will often write the parameter as a subscript, so the family is $\left(F_{\mu}\right)$, with $F_{\mu}: \mathbb{R}^{n} \rightarrow \mathbb{R}^{n}$.

Note that we could allow the regions themselves to vary with $\mu$ whilst retaining the same basic topology of the partition (neighbours etc), but provided these variations are sufficiently smooth, small and maintain this topology, then by a change of coordinates we may assume that the family has $\Sigma$ fixed. Thus every result below which assumes that $\left(F_{\mu}\right)$ is a continuous family of hybrid maps actually holds for any family $\left(G_{\mu}\right)$ of hybrid maps which, after a smooth $\mu$-dependent change of coordinates can be transformed into a continuous family. The restriction to continuous families as defined above makes the proofs considerably simpler, and, as noted, there is in fact no loss of generality in so doing.

Theorem 2. If $\left(F_{\mu}\right)$ is a continuous family of hybrid maps and $F_{0}$ has a regular snap-back repeller, then there is an open neighbourhood $\mathcal{M}$ of $\mu=0$ such that $F_{\mu}$ has a regular snap-back repeller for all $\mu \in \mathcal{M}$.

Proof: Note that standard hyperbolicity arguments using the implicit function theorem imply that if $x^{*}(0) \in R_{k}$ is a fixed point of $F_{0}$ and all the eigenvalues of the Jacobian matrix (with respect to $x$ ) lie outside the unit circle, then for all $\mu$ in a neighbourhood $\mathcal{N}_{0}$ of zero, $F_{\mu}$ will also have a continuously differentiable curve of fixed points $x^{*}(\mu) \in R_{k}$, and all the eigenvalues of the Jacobian matrix at $x^{*}(\mu)$ will also lie outside the unit circle.

Now consider the homoclinic orbit $\left(p_{r}(0)\right)$; we wish to show that there will be a homoclinic orbit to $x^{*}(\mu)$ for $\mu$ sufficiently close to zero. Since $F_{0}\left(p_{0}(0)\right)=x^{*}(0)$, the implicit function theorem again implies the existence of a unique, continuously differentiable solution $y=p_{0}(\mu)$ satisfying

$$
G_{0}(\mu, y)=F_{\mu}(y)-x^{*}(\mu)=0
$$

for $\mu \in \mathcal{N}_{1} \subset \mathcal{N}_{0}$ provided $D F_{0}\left(p_{0}(0)\right)$ is invertible. Moreover, by the continuity of $D F(x, \mu)$ in $\mu$ we may assume (restricting the size of $\mathcal{N}_{1}$ if necessary) that $D F\left(p_{0}(\mu), \mu\right) \neq 0$ for all $\mu \in \mathcal{N}_{1}$.

For $m \geq 1$, the implicit function theorem on

$$
G_{m}(\mu, y)=F_{\mu}(y)-p_{m-1}(\mu)=0
$$

with known solution $F_{0}\left(p_{m}(0)\right)=p_{m-1}(0)$, guarantees the existence of a unique continuously differentiable solution on an open neighbourhood $\mathcal{N}_{m+1} \subset \mathcal{N}_{m}$ of $\mu=0$ 
and on which $D F$ does not vanish. Thus for any finite set $\left(p_{r}(0)\right)_{0}^{N}$ there is an open neighbourhood $\mathcal{N}_{N+1}$ such that $p_{m}(\mu)$ is defined, close to $p_{m}(0)$ and $F_{\mu}\left(p_{m+1}(\mu)\right)=$ $p_{m}(\mu)$.

Of course, once $m$ is sufficiently large, $m>M^{\prime}$ say, $p_{m}(\mu)$ is close to $x^{*}(0)$ which is close to $x^{*}(\mu)$, and so the existence of the infinite set of points converging on $x^{*}(\mu)$ in backwards time with non-singular Jacobian follows directly from the nature of the fixed point, so we do not need to take infinite intersections of the $\mathcal{N}_{m}$ and can set $\mathcal{M}=\cap_{1}^{M^{\prime}} \mathcal{N}_{j}$.

This theorem establishes the persistence of a regular snap-back repeller. The next step is to describe the simplest bifurcations creating snap-back repellers which do not involve a change of stability of the fixed point $x^{*}$ itself.

\section{BifurCATION TO SNAP-BACK REPELLERS}

Suppose that $F_{\mu}$ is a continuous family of hybrid maps for $\mu$ in a neighbourhood $\mathcal{M}$ of $\mu=0$, and that $F_{0}$ has the following properties:

(i) there exist $k$ and $x_{0}^{*} \in R_{k}$ such that $F_{0}\left(x_{0}^{*}\right)=x_{0}^{*}$ and all the eigenvalues of $D F_{0}\left(x_{0}^{*}\right)$ lie strictly outside the unit circle;

(ii) there exists $m \geq 0$ and $p_{m}(0) \in \Sigma$, such that the intersection of $\Sigma$ with a small neighbourhood of $p_{m}(0)$ has a single connected component, and a sequence $\left(q_{n}\right) \in R_{i(m)}$ such that $\lim _{n \rightarrow \infty} q_{n}=p_{m}(0)$, and if $p_{m-1}(0)=\lim _{n \rightarrow \infty} F_{0}\left(q_{n}\right)$ then $p_{m-1}(0) \notin \Sigma$ and $p_{k-1}(0)=F_{0}\left(p_{k}\right)(0) \notin \Sigma, k=m-1, \ldots, 1$, with $F\left(p_{0}(0)\right)=x_{0}^{*}$ and $D F_{0}\left(p_{k}(0)\right)$ is non-singular, $k=0, \ldots, m-1$;

(iii) there exist $p_{r}(0) \in R_{i(r)}, r>m$, such that $p_{r}(0)=F\left(p_{r+1}(0)\right)$ and $\lim _{r \rightarrow \infty} p_{r}(0)=$ $x_{0}^{*}$ and $D F\left(p_{r}(0)\right)$ is non-singular.

Thus the points $\left(p_{r}(0)\right)$ almost satisfy the conditions for the existence of a snapback repeller, but the homoclinic orbit intersects the discontinuity set $\Sigma$ at a single point. Note that by the Implicit Function Theorem, (i) implies that there is an open neighbourhood $\mathcal{M}^{\prime}$ of zero and a continuously differentiable function $x^{*}: \mathcal{M}^{\prime} \rightarrow \mathbb{R}^{n}$ such that $x^{*}(\mu)$ is a repelling fixed point in $R_{k}$ for all $\mu \in \mathcal{M}^{\prime}$ and $x^{*}(0)=x_{0}^{*}$.

The final condition we impose ensures that the desired bifurcation to a regular snap-back repeller occurs as $\mu$ passes through zero:

(iv) if $\mu>0$ is sufficiently small then there exists $p_{m}(\mu) \in R_{i(m)}$ such that $F_{\mu}^{m}\left(p_{m}(\mu)\right)=x^{*}(\mu)$ and

$$
\lim _{\mu \downarrow 0} p_{m}(\mu)=p_{m}(0)
$$

and if $\mu<0$ then there is no point $x$ in $R_{i(m)}$ such that $F_{\mu}^{m}(x)=x^{*}(\mu)$. 
This condition could have been replaced by conditions on the derivatives of $F_{\mu}$ with respect to both $x$ and $\mu$ which would ensure these conditions using the Implicit Function Theorem again. The more transparent condition (iv) is preferred here, but it would usually be verified using the Implicit Function Theorem. The condition on $\mu<0$ could have been made less strong - it is enough that this holds locally to $p_{m}(0)$, but the additional restrictions on the neighbourhoods considered below create additional complications to the arguments which make the condition as stated more convenient, and we leave the minor generalization to local non-existence to the reader. A few simple consequences of these conditions are fairly immediate.

Lemma 3. Suppose that $\left(F_{\mu}\right)$ is a continuous family of hybrid maps which satisfies the conditions (i)-(iv) above. If $\mu>0$ is sufficiently small then $F_{\mu}$ has a regular snap-back repeller to the fixed point $x^{*}(\mu)$ containing a point close to $p_{m}(0)$ in $R_{i(m)}$. If $\mu<0$ then there is no homoclinic orbit to $x^{*}(\mu)$ which passes close to $p_{m}(0)$ in $R_{i(m)}$.

Before proving the main theorem (Theorem 6 below), we give a simple lemma which emphasizes the connection between maps of the interval and more general maps with snap-back repellers. The condition (28) in the Lemma 4 is precisely the condition for a topological horseshoe in one-dimensional dynamics; it is likely that variants of this lemma exist in the literature for expanding maps of $\mathbb{R}^{n}$, the novelty here is the interpretation in terms of snap-back repellers.

Lemma 4. Let $V_{i} \subset \mathbb{R}^{n}, i=1,2$, be closed, disjoint sets with non-empty interiors and $F$ a function from $\mathbb{R}^{n}$ to $\mathbb{R}^{n}$. If there exist positive integers $k_{i}$ such that $\left.F^{k_{i}}\right|_{V_{i}}$ is continuously differentiable, $i=1,2$, and

$$
V_{1} \cup V_{2} \subseteq F^{k_{i}}\left(V_{i}\right), \quad i=1,2
$$

and if all fixed points of $F^{k_{i}}$ in $V_{i}$ are repelling, then there exists a periodic point $x_{i}^{*}$ in $V_{i}$ such that there is a homoclinic orbit in $V_{1} \cup V_{2}$, biasymptotic to $x_{i}^{*}, i=1,2$.

Proof: Since $V_{j} \subset F^{k_{i}}\left(V_{i}\right)$, there exists a closed region $V_{i j} \subset V_{i}$ with non-empty interior such that $F^{k_{i}}\left(V_{i j}\right)=V_{j},\{i, j\} \in\{1,2\}^{2}$. By induction, for any $i_{1} \ldots i_{N} \in$ $\{1,2\}^{N}$ there exists $V_{i_{1} \ldots i_{N}} \subset V_{i_{1}}$ such that if $K_{r}=\sum_{1}^{r} k_{i_{r}}$

$$
F^{K_{r}}\left(V_{i_{1} \ldots i_{N}}\right) \subset V_{i_{r+1}}, r=1, \ldots, N-1 ; \quad F^{K_{N-1}}\left(V_{i_{1} \ldots i_{N}}\right)=V_{i_{N}} .
$$

Taking countable intersections, $V_{\mathbf{i}} \neq \emptyset$ for all $\mathbf{i} \in\{1,2\}^{\mathbb{N}}$.

Now, $V_{1^{\infty}}$ is non-empty and must contain a fixed point $x_{1}^{*} \in V_{1}$ of $F^{k_{1}}$; a periodic orbit for $F$, and by assumption this is repelling (so all eigenvalues of the Jacobian matrix of $F^{k_{1}}$ evaluated at $x_{1}^{*}$ lie outside the unit circle. Hence it has a local unstable manifold and there exists $R>0$ such that $V_{1^{R} j}$ lies in the local unstable manifold of $x_{1}^{*}$, i.e. there exist for all $x \in V_{1^{R} 2}$ there exists $\left(x_{n}\right)_{1}^{\infty}$ in $V_{1}$ with $x_{n} \rightarrow x_{1}^{*}$ such that $F^{k_{1}}\left(x_{i+1}\right)=x_{i} i=1,2, \ldots$ and $F^{k_{1}}\left(x_{1}\right)=x$. 
Since $F^{k_{2}}\left(V_{21}\right)=V_{1}$ there exists $p \in V_{2}$ such that $F^{k_{2}}(p)=x_{1}^{*}$. Similarly, there exists $x \in V_{1^{R_{2}}}$ such that $F^{k_{1}}(x)=p$. This, together with its preimages in $V_{1}$ provides the homoclinic orbit to $x_{1}^{*}$; the case for a homoclinic orbit to $x_{2}^{*} \in V_{2}$ is proved by interchanging the roles of the two subscripts.

This lemma has an obvious but interesting corollary about the existence of snapback repellers to periodic orbits.

Corollary 5. If $F$ has a regular snap-back repeller then it has infinitely many periodic orbits which are regular snap-back repellers, and infinitely many different regular snap-back repellers to the same periodic orbits.

Proof: Simply consider pairs of sets $B(j)$ (for large enough $j$ ) in the proof of Theorem 1 as the $V_{i}$ of the lemma.

Finally we are in a position to prove the main theorem, the creation of infinitely many snap-back repellers involving higher periods as part of the creation of a regular snap-back repeller.

Theorem 6. Let $\left(F_{\mu}\right)$ be a continuous family of hybrid maps satisfying properties (i)-(iv) above. Then there exists $L>0$ and a sequence $\left(\mu_{k}\right)_{L}^{\infty}$ with $\lim \mu_{k} \rightarrow 0$ such that if $\mu>\mu_{j}$ then $F_{\mu}$ has a regular snap-back repeller to a periodic orbit of period $j$.

Proof: Fix $\delta>0$ and et $U_{m}$ be a closed disc of radius $\delta$ around $p_{m}(0)$, and note that for $\mu>0$ sufficiently close to zero, $p_{m}(\mu) \in U_{m} \cap R_{i(m)}$. Let $\mathcal{M}$ be an open neighbourhood of zero such that for all $\mu \in \mathcal{M}, U_{m} \cap R_{i(m)}$ is a connected set with non-empty interior and let $D_{\mu}$ be a closed subset with non-empty interior with the property that it has preimages in the correct sets going backwards and forwards, which do not intersect boundaries, and the boundary of $D_{\mu}$ varies continuously with $\mu$ for $\mu \in \mathcal{M}$.

Now, choose $\mu_{m}<0<\mu_{M}$ in $\mathcal{M}$, and consider first $\mu=\mu_{m}$. By construction the image under $F^{m}$ of any subset of $R_{i(m)}$ containing $D_{\mu_{m}}$ which does not intersect $\Sigma$ does not contain $x^{*}\left(\mu_{m}\right)$, and hence contains a finite number (possibly zero) of the preimages of $D_{\mu_{m}}$. In particular there exists $L_{1}>0$ such that there can be no snap-back repellers involving orbits of period greater than $L_{1}$ close to $x^{*}\left(\mu_{m}\right)$.

Let $E_{\mu}=F_{\mu}^{m}\left(D_{\mu}\right)$. If $\mu<0, x^{*}(\mu) \notin E_{\mu}$, and hence $E_{\mu}$ contains a finite number (possibly zero) of the preimages of $D_{\mu}$. If $\mu>0$ then $x^{*}(\mu) \in E_{\mu}$ and hence $E_{\mu}$ contains an infinite number of these preimages. Let $j=L, \ldots$ label those preimages which correspond to periods greater than $L_{m}$ (and so which cannot have snap-back repellers if $\mu=\mu_{m}$ ) but which are in $E_{\mu_{M}}$. Applying Lemma 4 to pairs of these, 
establishes the existence of regular snap-back repellers of given sufficiently high periods, and then the robustness results show that there is a last time that these are created, providing the sequence $\left(\mu_{j}\right)_{L}^{\infty}$.

Note that if the eigenvalue of $D F_{\mu}\left(x^{*}(0)\right)$ with smallest modulus is real and positive then $\mu_{k}$ will accumulate typically on one side of $\mu=0$ and $\left|\mu_{r}\right|>\left|\mu_{r+1}\right|$; otherwise the convergence will typically be on both sides of $\mu=0$. This follows from the fact that if the eigenvalue is real and positive then the preimages typically converge to $x^{*}$ on one branch of the weakest unstable eigenvector of the Jacobian matrix.

As is the case with homoclinic tangencies in reversible systems, a simpler result holds for the creation of the periodic points which are the snap-back repellers in the previous theorem.

Corollary 7. Let $\left(F_{\mu}\right)$ be a continuous family of hybrid maps satisfying properties (i)-(iv) above. Then there exists $L>0$ and a sequence $\left(\tilde{\mu}_{k}\right)_{L}^{\infty}$ with $\lim \tilde{\mu}_{k} \rightarrow 0$ such that if $\tilde{\mu}>\tilde{\mu}_{j}$ then $F_{\mu}$ has a periodic orbit of period $j$; as $\tilde{\mu} \downarrow \tilde{\mu}_{j}$ one point on this orbit tends to $\Sigma$.

The proof is essentially exactly as in Theorem 6 except that we only need $E_{\mu}$ to contain a preimage of $D_{\mu}$ in order to have a fixed point of the appropriate iterate of the map. In the case of a real and positive weakest eigenvalue $\lambda_{u}$ a simple scaling argument suggests that

$$
\lim _{n \rightarrow \infty} \frac{\tilde{\mu}_{n+1}-\tilde{\mu}_{n}}{\tilde{\mu}_{n}-\tilde{\mu}_{n-1}}=\lambda_{u}^{-1}
$$

as in the case of saddlenode bifurcations near homoclinic tangencies [4] and a similar rate of convergence should be observed for the $\mu_{n}$ in Theorem 6 .

\section{Bifurcation for the Border-Collision Normal Form}

We return to our discussion of the bifurcations in the normal form (1) with a view to seeing how the results above apply there, and to confirm the expectations on the scaling likely to be observed.

To fix ideas we will consider

$$
T_{R}=7, \quad D_{R}=10, \quad D_{L}=-1 / 3, \quad T_{L}>0
$$

in which case (4) is automatically satisfied, $\lambda_{-}=2, \lambda_{+}=5$, and the condition (6) for the existence of a second preimage of the fixed point is

$$
1-10 T_{L}>0
$$

so the bifurcation occurs at $T_{L}=0.1$ with a regular snap-back repeller if $T_{L}<0.1$. Comparison with (18), (19) and (20) shows that at the bifurcation parameter, the preimages of $\left(x_{0}, y_{0}\right)$ converge to the fixed point tangential to the right hand branch of the weaker unstable direction, whilst the image of a neighbourhood of $\left(x_{0}, y_{0}\right)$ in 


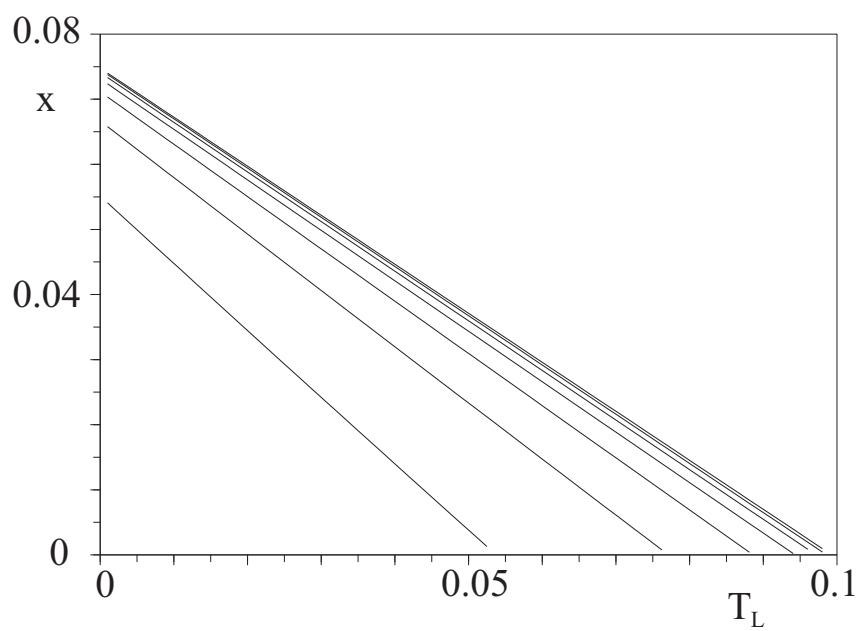

Figure 2. Locus of periodic points which are involved in border collision bifurcations close to $T_{L}=0.1$ The $x$-coordinate is shown as a function of $T_{L}$ in the $\left(T_{L}, x\right)$ plane. The points are on orbits with codes $R L R^{k+2}$, with, from left to right, $k=2,3,4,5,6,7,8$.

$x>0$ lies to the left of the fixed point. In particular, we expect there to be no bifurcations before the creation of the regular snap-back repeller; all the bifurcations associated with the creation of this repeller will be in $T_{L}<0.1$.

Thinking of $\nu=1-10 T_{L}$ as the natural bifurcation parameter, dictating how far to the right of the fixed points iterates can reach, and elementary consideration of the geometric convergence of the preimages on the right hand branch of the fixed point at a rate $\lambda_{-}^{-1}$ shows that the creation of new periodic orbits with codes $R L R^{n}$ by border collisions will occur at parameters $\tilde{\nu}_{n}$ which tend to zero from above with scaling given by (29), or put another way

$$
\tilde{\nu}_{n} \sim K \lambda_{-}^{-n}=K 2^{-n} \text { as } n \rightarrow \infty
$$

Figure 5 shows the creation of some of these orbits, whilst Table 1 provides strong evidence for the scaling results expected.

As shown earlier, each of these orbits become snap-back repellers in their own right as $T_{L}$ decreases.

\section{Conclusion}

The snap-back repeller is the natural extension of homoclinic orbits to hybrid non-invertible maps with repelling regions. Their existence provides a proof of the existence of a chaotic set. In this paper we have established a definition of regular 
TABLE 1. Parameter values for the creation of periodic orbits $\left(T_{k}\right.$ is the value of $T_{L}$ at which an orbits with code $R L R^{2+k}$ is created by border collision) together with confirmation of the general scaling (29) and a version of (32) with a final column indicating that this is converging.

$\begin{array}{ccccc}k & T_{k} & \frac{T_{k+1}-T_{k}}{T_{k}-T_{k-1}} & u_{k}=2^{k-1}\left(1-10 T_{k}\right) & u_{k} / u_{k+1} \\ & & & & \\ 1 & 0.0059601686 & & 0.9403983 & 1.0167351 \\ 2 & 0.05375401694 & 0.4888559 & 0.9249197 & 1.0105461 \\ 3 & 0.07711831980 & 0.4926944 & 0.9152672 & 1.0062112 \\ 4 & 0.08862978210 & 0.4955881 & 0.9096174 & 1.0035012 \\ 5 & 0.09433472620 & 0.4974720 & 0.9064438 & 1.0019146 \\ 6 & 0.09717277619 & 0.4986025 & 0.9047116 & 1.0010245 \\ 7 & 0.09858783488 & 0.4992467 & 0.9037857 & 1.0005396 \\ 8 & 0.09929429821 & 0.4996012 & 0.9032983 & 1.0002808 \\ 9 & 0.09964724816 & 0.4997917 & 0.9030447 & 1.0001449 \\ 10 & 0.09982364962 & 0.4998923 & 0.9029139 & 1.0000742 \\ 11 & 0.09991183136 & 0.4999456 & 0.9028469 & 1.0000399 \\ 12 & 0.09995591743 & & 0.9028109 & \end{array}$

snap-back repellers which implies persistence of the snap-back repeller under perturbation of the system. This has made it possible to described one mechanism by which such a regular snap-back repeller comes into existence, and some of the bifurcation phenomena associated with this. In particular we find an infinite sequence of bifurcations creating more complicated snap-back repellers associated with periodic orbits which converge to the fixed point. These bifurcations are analogous to similar cascades in the standard theory of homoclinic tangencies in invertible systems. A motivating example from bifurcation theory was used to show how the more general mechanism described arises in border-collision bifurcations.

Acknowledgements: This work was partially funded by EPSRC Grant No. EP/E050441/1.

\section{REFERENCES}

[1] S. Banerjee and C. Grebogi. Border collision bifurcations in two-dimensional piecewise smooth maps. Phys. Rev. E, 59:4052-4061, 1999.

[2] S. Banerjee, J. A. Yorke, and C. Grebogi. Robust chaos. Phys. Rev. Lett., 80:3049-3052, 1998.

[3] A. Boyarsky, P. Góra, and V. Lioubimov. Snap-back repellers and scrambled sets in general topological spaces. Nonlinear Anal., 43:591-604, 2001.

[4] P. Gaspard and X. J. Wang. Homoclinic orbits and mixed-mode oscillations in far-fromequilibrium systems. J. Stat. Phys., 48:151-199, 1987. 
[5] N. K. Gavrilov and L. P. Shilnikov. On three-dimensional dynamical systems close to systems with a structurally unstable homoclinic curve. i. Math. USSR Sb., 17:467-485, 1972.

[6] N. K. Gavrilov and L. P. Shilnikov. On three-dimensional dynamical systems close to systems with a structurally unstable homoclinic curve. ii. Math. USSR Sb., 19:139-156, 1973.

[7] P. Glendinning and C. H. Wong. Border collision bifurcations, snap-back repellers and chaos. Phys. Rev. E, 79:025202, 2009.

[8] J. Guckenheimer and P. J. Holmes. Nonlinear oscillations, dynamical systems, and bifurcations of vector fields. Springer-Verlag, New York, 1983.

[9] C. Li and G. Chen. An improved version of the marotto theorem. Chaos Solit. Fract., 18:69-77, 2003.

[10] W. Lin and G. Chen. Heteroclinical repellers imply chaos. Int. J. Bif. \& Chaos, 16:1471-1489, 2006.

[11] W. Lin, J. Wu, and G. Chen. Generalized snap-back repeller and semi-conjugacy to shift operators of piecewise continuous transformations. Disc. Cont. Dyn. Syst. A, 19:103-119, 2007.

[12] F. R. Marotto. Snap-back repellers imply chaos in rn. J. Math. Anal. Appl., 63:199-223, 1978.

[13] F. R. Marotto. On refefining a snap-back repeller. Chaos, Solit. Fract., 25:25-28, 2005.

[14] H. E. Nusse and J. A. Yorke. Border-collision bifurcation including 'period two to period three' for piecewise smooth systems. Physica D, 57:39-57, 1992.

[15] Y. Shi and P. Yu. Chaos induced by regular snap-back repellers. J. Math. Anal. Appl., 337:14801494, 2008. 